\title{
Magnetic Vortex Nanodiscs Enable Remote Magnetomechanical Neural Stimulation
}

Danijela Gregurect,f,\#\#, Alexander W. Senkot,,,\#\#, Andrey Chuvilin||,\#, Pooja D. Reddy§, Ashwin Sankararamant, Dekel Rosenfeld $\dagger, \neq$, Po-Han Chiangt,‡, Francisco Garciał,, Ian

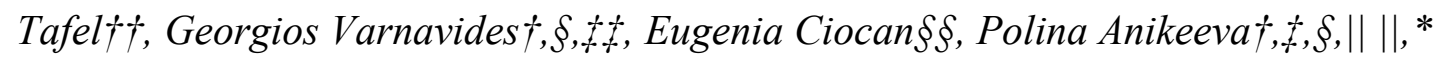

$\dagger$ Research Laboratory of Electronics, Massachusetts Institute of Technology, Cambridge, Massachusetts, 02139, USA

\$ McGovern Institute for Brain Research, Massachusetts Institute of Technology, Cambridge, Massachusetts, 02139, USA

$\S$ Department of Materials Science and Engineering, Massachusetts Institute of Technology, Cambridge, Massachusetts, 02139, USA

||CIC nanoGUNE, E20018, San Sebastián, Spain

\# Ikerbasque, Basque Foundation for Science, 48013, Bilbao, Spain

$\dagger \dagger$ Department of Neurosurgery, Brigham and Women's Hospital, Boston, Massachusetts, 02115, USA

\$\$ John A. Paulson School of Engineering and Applied Sciences, Harvard University, Cambridge, Massachusetts, 02138, USA

$\S \S$ Department of Engineering and Physical Sciences, Bunker Hill Community College, Boston, Massachusetts, 02129, USA

|| || Department of Brain and Cognitive Sciences, Massachusetts Institute of Technology, Cambridge, Massachusetts, 02139, USA 


\section{Magnetic Forces Calculations}

In the presence of static or slowly varying magnetic fields magnetic nanodiscs (MNDs) can transduce tension to cell membranes according to three mechanisms: (1) torque; (2) inter-particle attractive forces; and (3) gradient force. Below we use simple analytical models to calculate relative contributions from these three mechanisms and identify the most likely underlying principle for magnetomechanical control of cell calcium.

\section{Moment of an individual MND:}

The average volume of a MND used in this study is:

$V \approx \pi r^{2} h=\pi \times\left(113 \times 10^{-9} \mathrm{~m}\right)^{2} \times\left(37 \times 10^{-9} \mathrm{~m}\right)=1.48 \times 10^{-21} \mathrm{~m}^{3}$.

The moment of a uniformly magnetized magnetite particle with that volume is:

$$
|\vec{\mu}|=\mathrm{V} \cdot \rho \cdot \mathrm{M}_{s}=1.48 \times 10^{-21} \mathrm{~m}^{3}\left(\frac{5150 \mathrm{~kg}}{\mathrm{~m}^{3}}\right)\left(\frac{83 \mathrm{Am}^{2}}{\mathrm{~kg}}\right)=6.3 \times 10^{-16} \mathrm{Am}^{2}
$$

where $\rho=5150 \mathrm{~kg} / \mathrm{m}^{3}$ and $M_{s}=83 \mathrm{Am}^{2} / \mathrm{kg}$ are the density and saturation magnetization of magnetite.

\section{Torque:}

The torque on a magnetic dipole in a uniform magnetic field is given by: ${ }^{1}$

$$
\vec{\tau}=\vec{\mu} \times \vec{B}
$$

where $\vec{\mu}$ is the dipole moment and $\vec{B}$ is the magnetic field.

Then the torque magnitude is:

$$
|\vec{\tau}|=|\vec{\mu}||\vec{B}| \sin \theta_{\vec{\mu}-\vec{B}}
$$

The maximum case occurs when the angle between the magnetic moment and the applied field $\left(\theta_{\vec{\mu}-\vec{B}}\right)$ is 90 degrees, in which case:

$$
|\vec{\tau}|=|\vec{\mu}||\vec{B}|
$$

For a magnetic field of $26 \mathrm{mT}$ and a uniformly magnetized MND:

$$
|\vec{\tau}|=6.3 \times 10^{-16} \mathrm{Am}^{2} \times 0.026 \mathrm{~T}=1.6 \times 10^{-17} \mathrm{Nm}
$$

The force on the edge of a disk to oppose this magnetic torque scales inversely with the disk radius, so for a $226 \mathrm{~nm}$-diameter disk, that force is:

$$
F=1.4 \times 10^{-10} \mathrm{~N}
$$


which is almost three orders of magnitude greater than the force required to activate a mechanosensitive ion channel $(\sim 0.3 \mathrm{pN}) .{ }^{2}$ This analysis provides an upper bound for the force as it assumes that the MND would be completely magnetized in-plane as well as that the MND dipole moment is perpendicular to the field.

A MND 10 degrees off from the applied field would be $\sim 80 \%$ magnetized, ${ }^{3}$ and the resulting force would be $18 \mathrm{pN}$, which is still significantly greater than the response threshold of mechanoreceptors, and thus torque on individual MNDs is a plausible mechanism for magnetomechanical stimulation.

\section{Inter-particle attractive forces}

For two magnetic dipoles $\vec{\mu}_{1}$ and $\vec{\mu}_{2}$ that are magnetized along the $x$-axis, and which are also separated by a distance $x$ along the $\mathrm{x}$-axis, the attraction force is:

$$
\vec{F}=\frac{3 \mu_{0}}{2 \pi} \frac{\left|\vec{\mu}_{1}\right|\left|\vec{\mu}_{2}\right|}{x^{4}} \hat{x}
$$

If two MNDs are fully magnetized in the $x$-direction (with their easy axes along the $x$-direction) and separated by $1 \mu \mathrm{m}$, the magnitude of the attraction force is

$$
|\vec{F}|=\frac{3 \times\left(1.26 \times 10^{-6} \frac{\mathrm{kg} \mathrm{m}}{\mathrm{s}^{2} \mathrm{~A}^{2}}\right)}{2 \pi} \frac{\left(6.3 \times 10^{-16} \mathrm{Am}^{2}\right)^{2}}{\left(10^{-6} \mathrm{~m}\right)^{4}}=2.4 \times 10^{-13} \mathrm{~N}
$$

This is approximately the force required to activate mechanosensitive ion channels, and is thus also a possible mechanism for the method of magnetomechanical stimulation.

\section{Gradient force:}

The gradient force acting on a magnetic moment in a magnetic field is: ${ }^{1}$

$$
\vec{F}=\nabla(\vec{\mu} \cdot \vec{B})
$$

In the case of a magnetic dipole with its moment in the direction of the gradient of $\vec{B}$, the magnitude of the gradient force is:

$$
|\vec{F}|=|\vec{\mu}| \frac{d \vec{B}}{d x} \mid
$$

In the case of a MND in the magnetic field simulated in Figure S9, that force is equal to

$$
|\vec{F}|=\left(6.3 \times 10^{-16} A m^{2}\right) \times(0.43 \mathrm{~T} / \mathrm{m})=2.7 \times 10^{-16} \mathrm{~N}
$$


This is three orders of magnitude lower than the $0.3 \mathrm{pN}$ activation threshold for the mechanoreceptors. Large ( $\sim 2 \mu \mathrm{m}$ diameter) aggregates would be necessary to achieve the forces required to trigger mechanosensitive channels. While such aggregates can in principle occur, our dynamic light scattering spectra of MND solutions, SEM images of dorsal root ganglia (DRGs) decorated with MNDs, and bright field images of DRGs decorated with MNDs do not indicate prevalence of large clusters. Consequently, gradient force is unlikely to be effective for magnetomechanical modulation in large volumes such as in our experiment.

\section{Magnetic Energy Calculations}

In addition to questioning whether MNDs can produce significant forces in the AMF conditions used in this work, it is also worth considering whether they can transduce an amount of energy comparable to the amount required to trigger a mechanosensitive ion channel. For PIEZO1, a known mechanosensor, that energy is $\sim 40 \mathrm{k}_{\mathrm{B}} \mathrm{T}$, ${ }^{4}$ where $\mathrm{k}_{\mathrm{B}}$ is the Boltzmann constant, $\mathrm{T}$ is the temperature in Kelvin, and $\mathrm{k}_{\mathrm{B}} \mathrm{T}$ is approximately $4 \times 10^{-21} \mathrm{~J}$ at room temperature.

\section{Zeeman Energy}

The potential energy of a magnetic moment in a magnetic field is: ${ }^{1}$

$$
U=-\vec{\mu} \cdot \vec{B}
$$

For a MND with its moment aligned with a $26 \mathrm{mT}$ magnetic field, that is

$$
U=-\left(6.3 \times 10^{-16} \mathrm{Am}^{2}\right)(0.026 \mathrm{~T})=-1.6 \times 10^{-17} \mathrm{~J}=4.0 \times 10^{3} k_{B} T
$$

This represents the kinetic energy that a MND can produce by rotating from perpendicular to parallel with the applied field, and it is 100x greater than the gating energy of PIEZO1.

\section{Shape Anisotropy Energy}

A relevant question is whether a MND will physically rotate, or whether its moment will align with the field independent of the particle orientation. To find out, we can consider the shape anisotropy energy, which in this case is the energetic cost of the moment being out of the plane of the MND.

If we approximate a MND as an oblate spheroid with axes $\mathrm{a}<\mathrm{b}=\mathrm{c}$ and $\mathrm{c} / \mathrm{a}=\mathrm{m}$, we can calculate its demagnetizing factors: ${ }^{5}$ 


$$
\begin{gathered}
N_{C}=N_{B}=\frac{1}{2\left(m^{2}-1\right)}\left(\frac{m^{2}}{\sqrt{m^{2}-1}} \arcsin \left(\frac{\sqrt{m^{2}-1}}{m}\right)-1\right) \\
N_{A}=1-2 N_{C}
\end{gathered}
$$

For a MND of diameter $226 \mathrm{~nm}$ and thickness $37 \mathrm{~nm}, \mathrm{~m}=6.1$.

$$
\begin{gathered}
N_{C}=N_{B}=\frac{1}{2\left(6.1^{2}-1\right)}\left(\frac{6.1^{2}}{\sqrt{6.1^{2}-1}} \arcsin \left(\frac{\sqrt{6.1^{2}-1}}{6.1}\right)-1\right)=0.11 \\
N_{A}=1-2 \times 0.11=0.78
\end{gathered}
$$

If we use the equation for the shape anisotropy of a uniaxial particle, we can estimate the energy difference between in-plane and out-of-plane magnetization of a MND (per unit volume):

$$
\begin{aligned}
\Delta E_{\text {shape }} & =\frac{1}{2} \mu_{0} \Delta N M_{s}^{2}\left[\sin ^{2} \theta_{1}-\sin ^{2} \theta_{2}\right] \\
& =\frac{1}{2}\left(1.26 \times 10^{-6} \frac{\mathrm{kg} m}{\mathrm{~s}^{2} \mathrm{~A}^{2}}\right)(0.78-0.11)\left(438 \times 10^{3} \mathrm{~A} / \mathrm{m}\right)^{2}\left[\sin ^{2}\left(\frac{\pi}{2}\right)-\sin ^{2}(0)\right] \\
& =8.1 \times 10^{4} \frac{\mathrm{J}}{\mathrm{m}^{3}}
\end{aligned}
$$

Per MND, the shape anisotropy energy is:

$$
E_{\text {shape }}=\left(8.1 \times 10^{4} \frac{\mathrm{J}}{\mathrm{m}^{3}}\right)\left(1.48 \times 10^{-21} \mathrm{~m}^{3}\right)=1.2 \times 10^{-16} \mathrm{~J}=2.9 \times 10^{4} \mathrm{k}_{B} T
$$

This energy is an order of magnitude greater than the Zeeman energy, indicating that the moment is trapped in the in-plane orientation and the MND must physically rotate to minimize its Zeeman energy.

\section{Inter-Particle Interaction Energy}

The interaction energy of two magnetic dipoles separated by a displacement $\hat{r}$ is ${ }^{1}$ :

$$
U=\frac{\mu_{0}}{4 \pi} \frac{1}{r^{3}}\left[\vec{\mu}_{1} \cdot \vec{\mu}_{2}-3\left(\vec{\mu}_{1} \cdot \hat{r}\right)\left(\vec{\mu}_{2} \cdot \hat{r}\right)\right]
$$

In the simple case when the moments are oriented in the $\hat{x}$ direction and also separated along the $\hat{x}$ direction, this reduces to:

$$
U=-\frac{\mu_{0}}{2 \pi} \frac{1}{r^{3}}\left|\vec{\mu}_{1}\right|\left|\vec{\mu}_{2}\right|
$$

Plugging in for two MNDs one micron apart,

$$
U=-\frac{1.26 \times 10^{-6} \frac{\mathrm{kg} \mathrm{m}}{\mathrm{s}^{2} \mathrm{~A}^{2}}}{2 \pi} \frac{1}{\left(10^{-6} \mathrm{~m}\right)^{3}}\left(6.3 \times 10^{-16} \mathrm{Am}^{2}\right)^{2}=8.0 \times 10^{-20} \mathrm{~J}=19 \mathrm{k}_{B} \mathrm{~T}
$$


For two MNDs $500 \mathrm{~nm}$ apart,

$$
U=-\frac{1.26 \times 10^{-6} \frac{\mathrm{kg} \mathrm{m}}{\mathrm{s}^{2} \mathrm{~A}^{2}}}{2 \pi} \frac{1}{\left(5 \times 10^{-7} \mathrm{~m}\right)^{3}}\left(6.3 \times 10^{-16} \mathrm{Am}^{2}\right)^{2}=6.4 \times 10^{-19} \mathrm{~J}=155 \mathrm{k}_{B} \mathrm{~T}
$$

Thus, two MNDs moving from $1 \mu \mathrm{m}$ to $500 \mathrm{~nm}$ apart will produce 3.4 times as much energy as is required for the gating of PIEZO1, and inter-particle attraction is a plausible mechanism for magnetomechanical stimulation.

\section{Energy of a MND in a magnetic field gradient}

The kinetic energy produced by a MND moving $1 \mu \mathrm{m}$ down a $0.4 \mathrm{~T} / \mathrm{m}$ field gradient is $2.5 \times 10^{-22} \mathrm{~J}=0.06 k_{B} T$. This means that 652 MNDs moving together would produce enough energy to trigger PIEZO1. It is the least energetic MND-field interaction considered and is an unlikely mechanism for magnetomechanical stimulation.

\section{Supplementary References}

1. Griffiths, D. J. Magnetic Fields in Matter. In Introduction to Electrodynamics, 4th ed. Cambridge University Press: Cambridge, United Kingdom, 2017; pp 267-292.

2. Howard, J.; Hudspeth, A. J. Compliance of the Hair Bundle Associated with Gating of Mechanoelectrical Transduction Channels in the Bullfrog's Saccular Hair Cell. Neuron 1988, $1,189-199$.

3. Yong, Y.; Xiaoli, L.; Yunbo, L.; Seng, H. T.; Xianhui, X.; Weixing, X.; Taishi, Z.; Jie, F.; Wen, X.; Jun, D. Orientation Mediated Enhancement on Magnetic Hyperthermia of Fe3O4 Nanodisc. Adv. Funct. Mater. 2015, 25, 812-820.

4. Cox, C. D.; Bae, C.; Ziegler, L.; Hartley, S.; Nikolova-Krstevski, V.; Rohde, P. R.; Ng, C.A.; Sachs, F.; Gottlieb, P. A.; Martinac, B. Removal of the Mechanoprotective Influence of the Cytoskeleton Reveals Piezo1 Is Gated by Bilayer Tension. Nat. Commun. 2016, 7, 10366.

5. Cullity, B. D.; Graham, C. D. Experimental Methods. In Introduction to Magnetic Materials, 2nd ed. John Wiley \& Sons: Piscataway, NJ, 2009; pp 54. 
Supplementary Figures
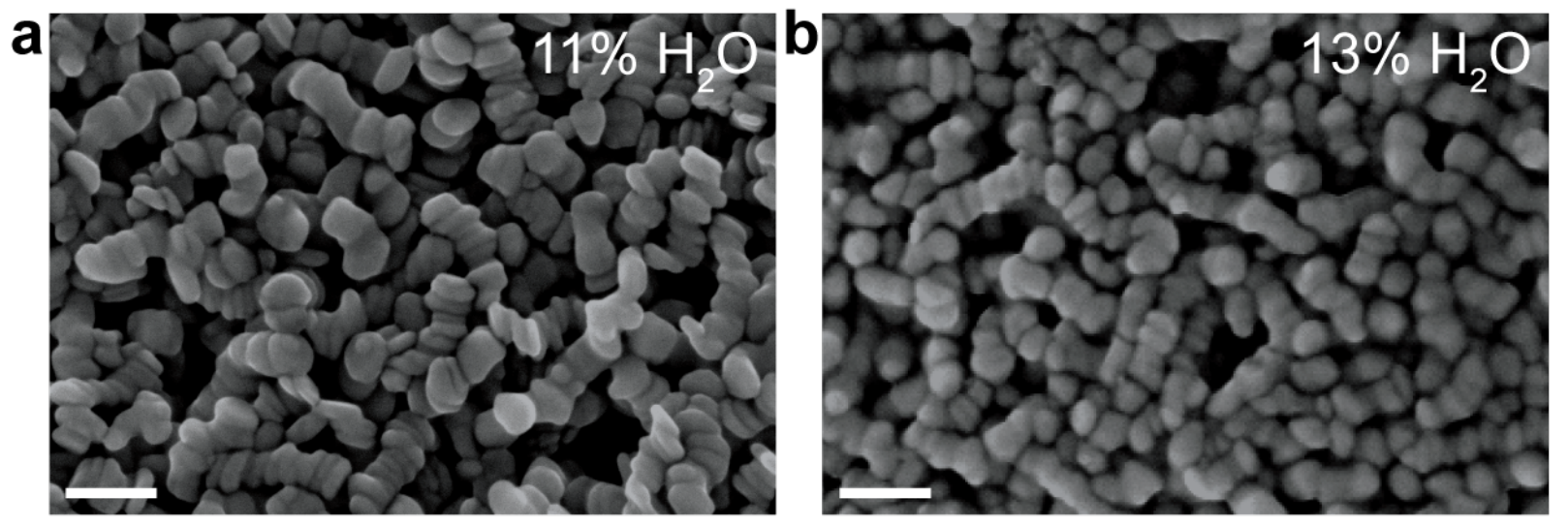

Supplementary Figure 1: Scanning electron micrographs of magnetite particles from highwater-content solvothermal synthesis. Magnetite nanoparticles synthesized in a two-step reaction in which the first (solvothermal) step had $\mathbf{a}, 11 \% \mathrm{H}_{2} \mathrm{O}$ and $\mathbf{b}, 13 \% \mathrm{H}_{2} \mathrm{O}$ in the reaction mixture. Scale bars $=200 \mathrm{~nm}$. 

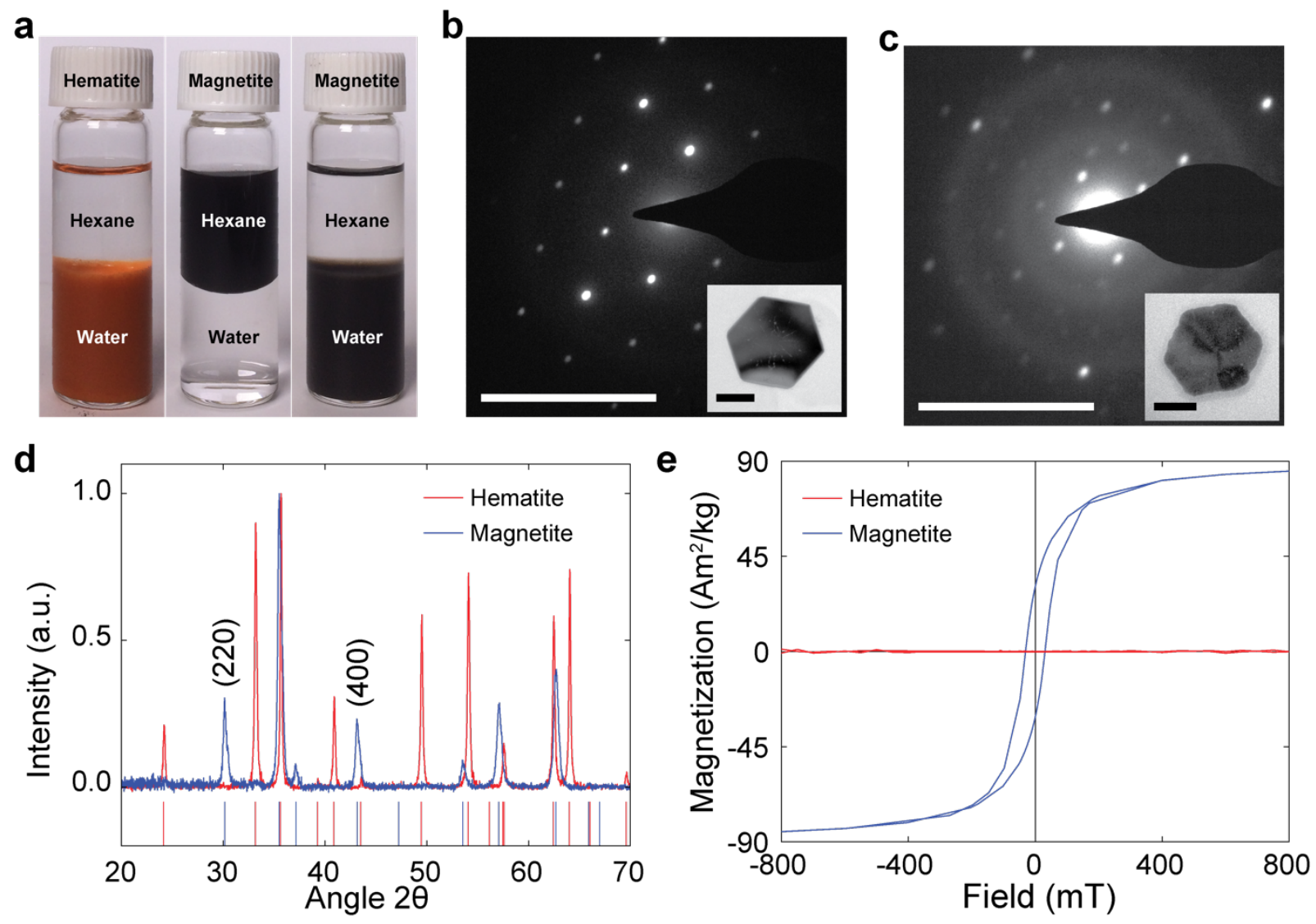

Supplementary Figure 2: Hematite to magnetite conversion. a, Hematite nanodiscs are synthesized in aqueous solution, then reduced to magnetite and at the same time coated with oleic acid (making them hydrophobic), then coated with the amphiphilic polymer PMAO to transfer them back into water. Note the color change upon reduction. b,c, Single particle electron diffraction patterns and transmission electron microscope images (insets) of hematite (b) and magnetite (c) nanodiscs. Note the hexagonal single crystal pattern in (b) and the polycrystalline cubic pattern in (c). Scale bars $=100 / \mathrm{nm}$ and $100 \mathrm{~nm}$ (inset). d, Powder X-ray diffraction data for hematite and magnetite nanodiscs, indicating the complete conversion of their structure upon reduction. e, Magnetization curves for hematite (red) and magnetite (blue) resulting from vibrating sample magnetometry (VSM). 
a

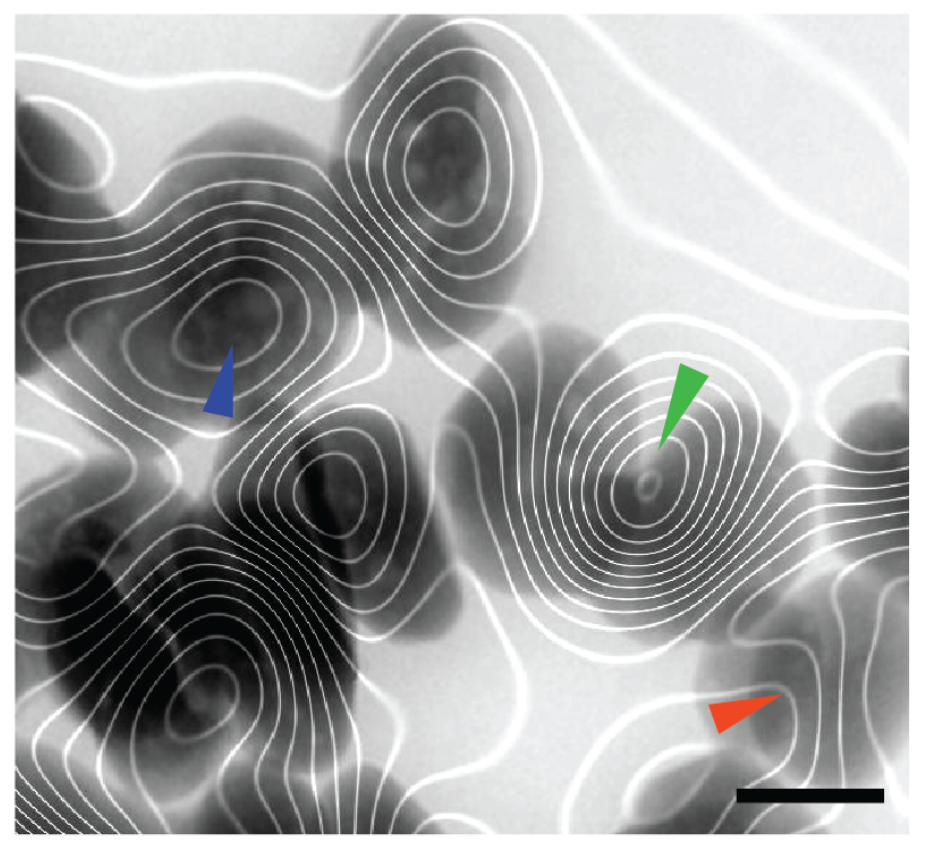

b

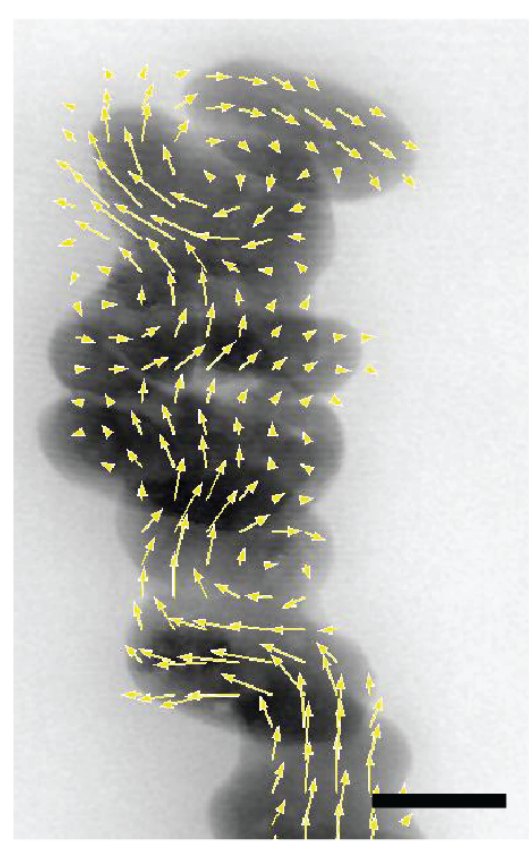

Supplementary Figure 3: Electron holography performed on clustered MNDs. a, Magnetic field lines in aggregated neighboring and overlapping MNDs. Blue arrow indicates vortex alignment in one MND. Green arrow marks formation of vortex center between two MNDs. Red arrow points to semi parallel field lines in the MND. b, Magnetic spins are partially aligned parallel to the stack axis of stacked MNDs. Scale bars $=100 \mathrm{~nm}$. 


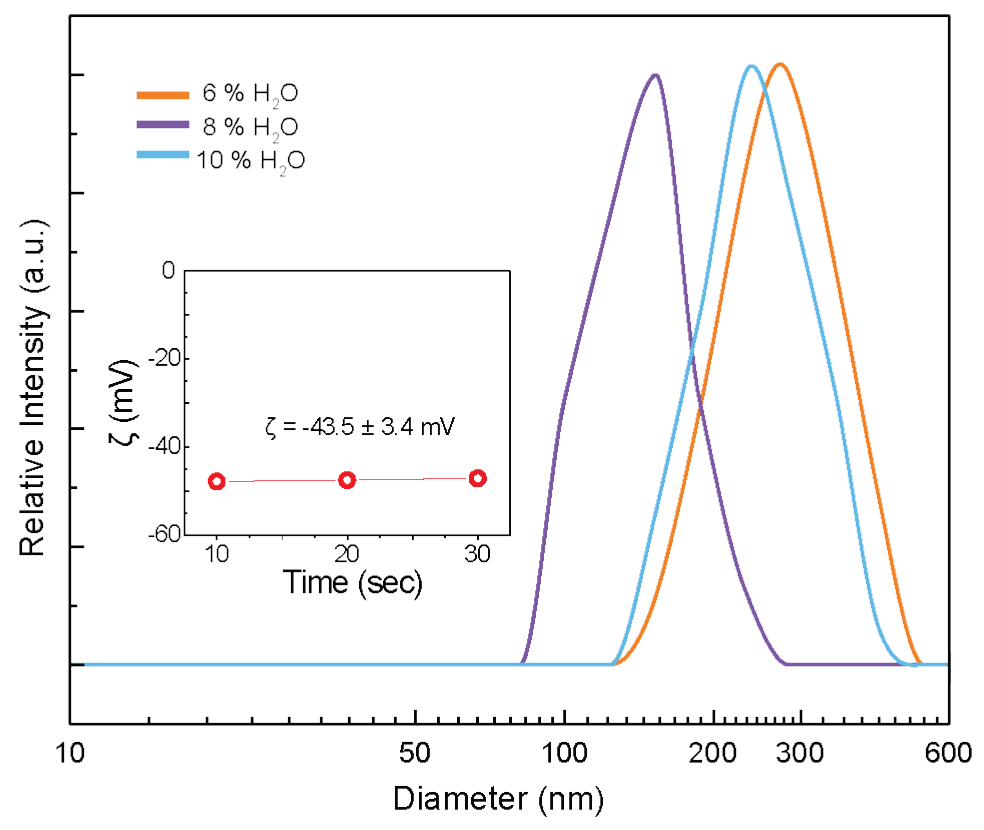

Supplementary Figure 4: Hydrodynamic size and zeta potentials of MNDs. Hydrodynamic size measurements for MNDs synthesized with $6 \% \mathrm{H}_{2} 0,8 \% \mathrm{H}_{2} 0$ and $10 \% \mathrm{H}_{2} 0$, and coated with PMAO show larger average diameters compared to dry, non-coated MNDs obtained from electron microscopy analysis. A negative zeta potential of $-43.5 \mathrm{mV}$ (shown in inset) results from charged carboxylic groups of PMAO.
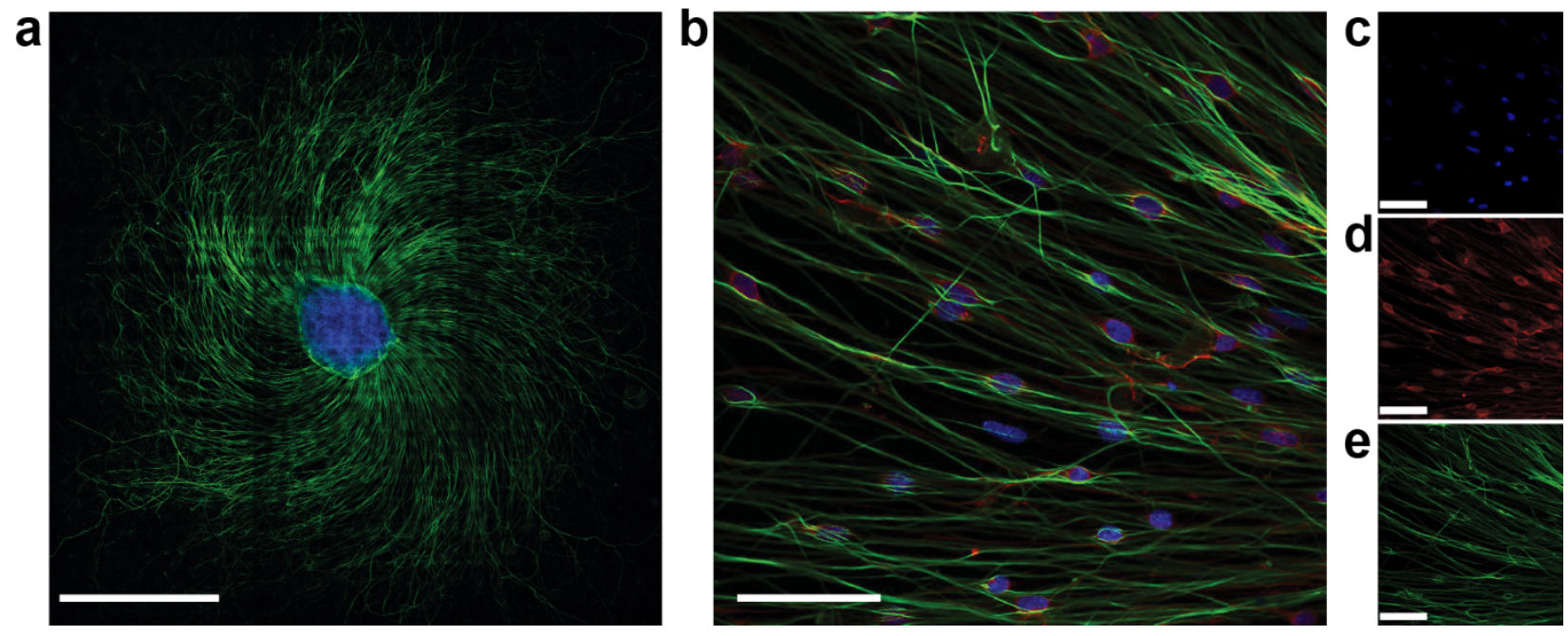

Supplementary Figure 5: Confocal microscope images of dorsal root ganglion (DRG) explant. a, Mosaic image of whole DRG explant stained for neurofilament (green) and DAPI (blue). Scale bar $=500 \mu \mathrm{m}$. b-e, Confocal microscope image of DRG outgrowth stained for cytoskeleton (Neurofilament, green), Schwann cells (S100, red) and nuclei (DAPI, blue). Scale bars $=50 \mu \mathrm{m}$. 


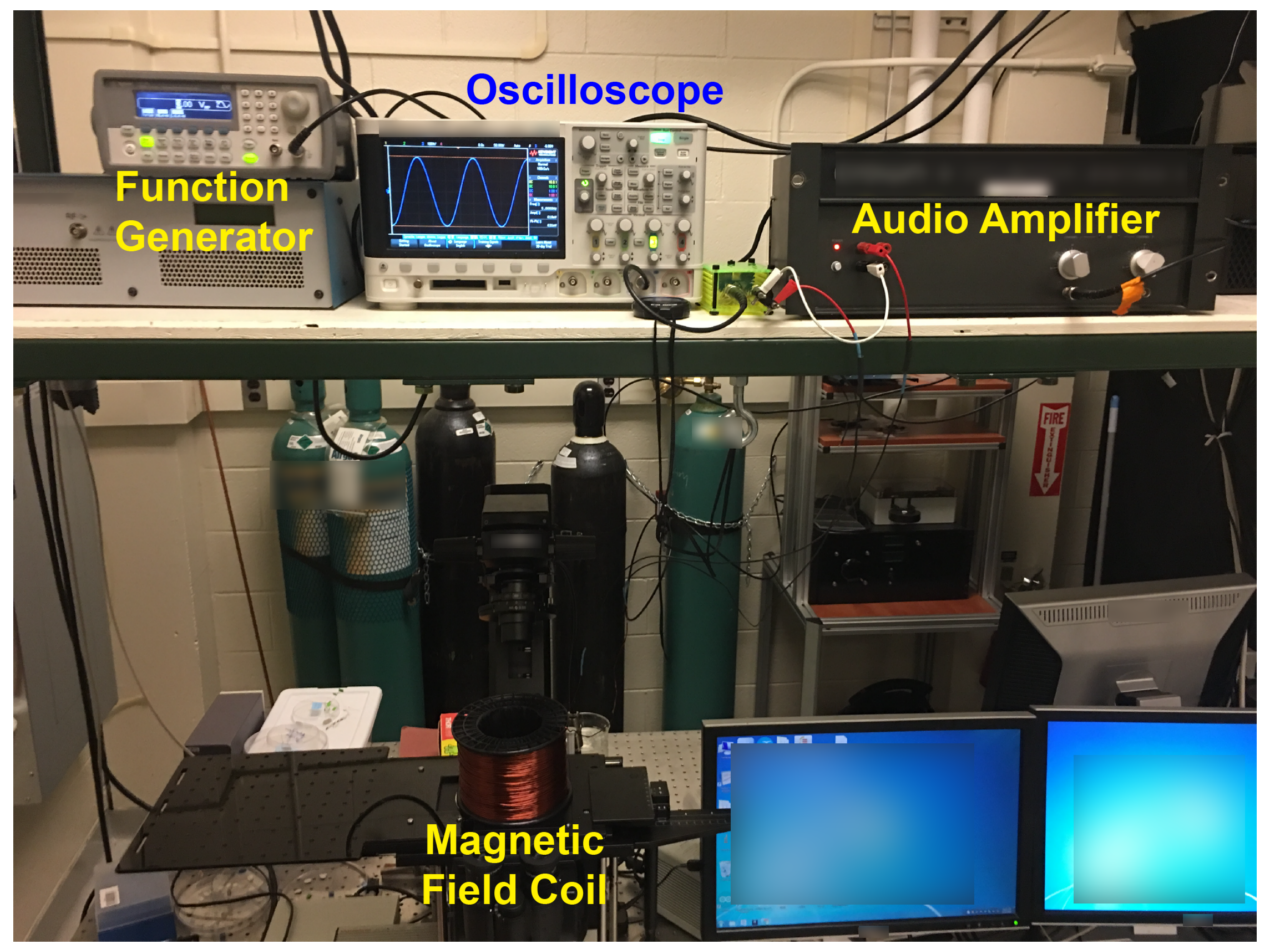

Supplementary Figure 6: Custom-built magnetic setup. $\mathrm{Ca}^{2+}$ imaging in an inverted fluorescence microscope with in situ magnetomechanical stimulation via copper wire solenoid, controlled with a function generator, oscilloscope, shunt resistor and $300 \mathrm{~W}$ amplifier. 

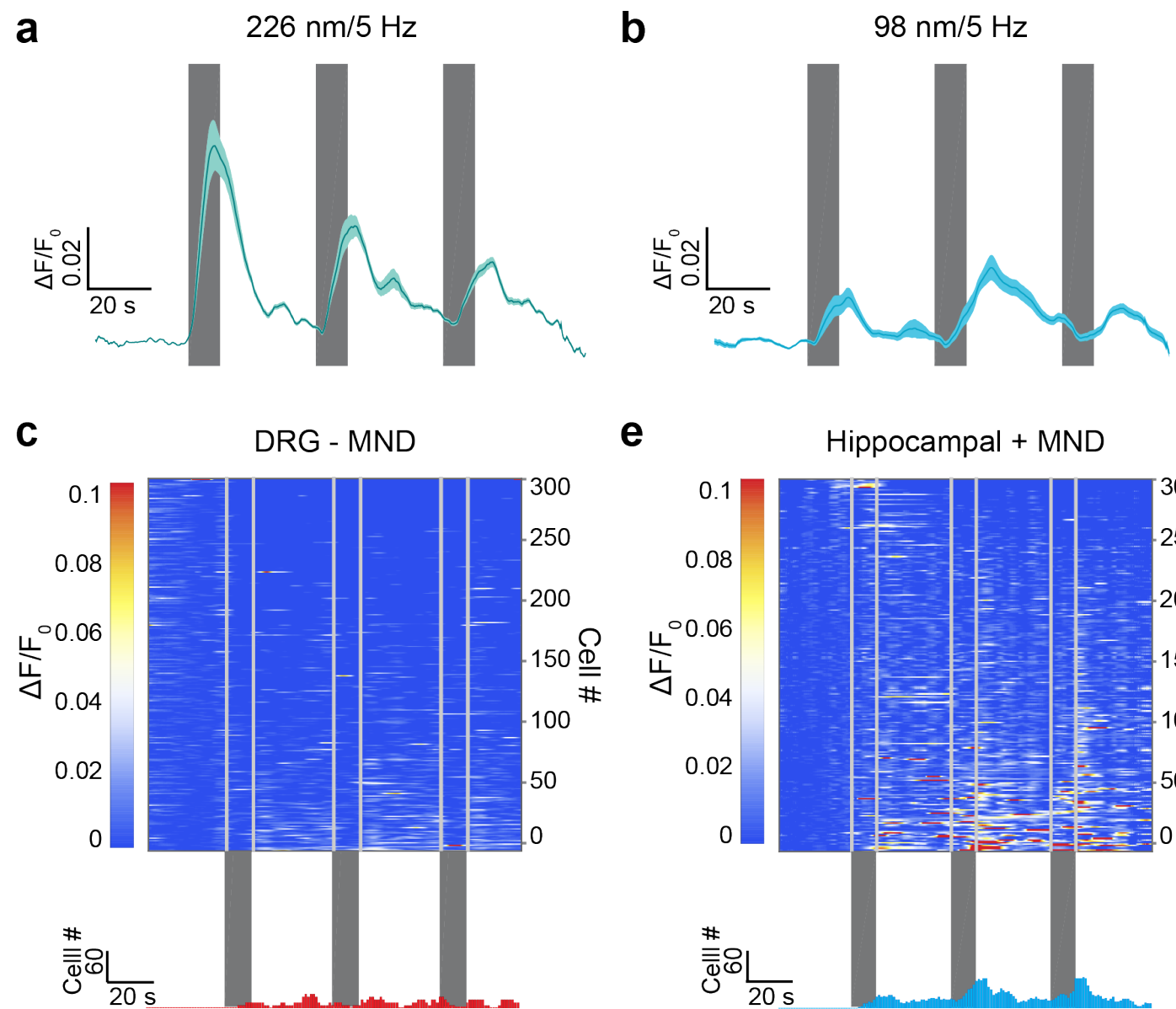

e
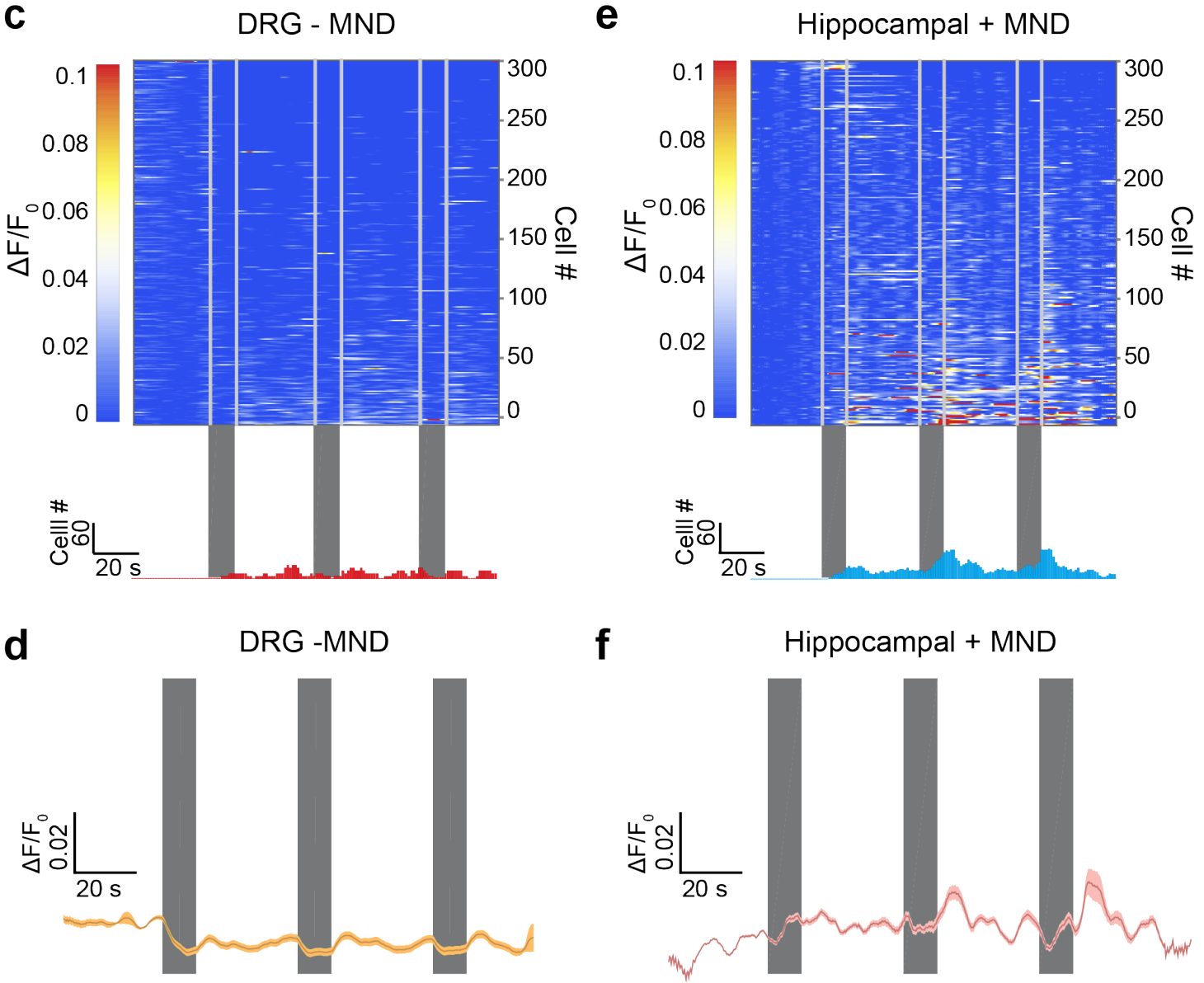

Supplementary Figure 7: Fluorescence traces resulting from $\mathrm{Ca}^{2+}$ imaging for MNDs and controls. a, Fluorescence trace corresponding to main Figure 4f. DRGs are incubated with $226 \mathrm{~nm}$ diameter MNDs. b, Fluorescence trace for calcium influx of DRGs decorated with MNDs of $98 \mathrm{~nm}$ diameter corresponding to main Figure 4g. c,d, No cell activity observed when MF is applied to the DRGs without MNDs. e,f, Activity of hippocampal neurons decorated with $226 \mathrm{~nm}$ MNDs when MF is applied. This activity is significantly lower compared to the activity of DRGs. Bar graph and significance levels are shown in main Figure $4 \mathrm{~h} .10 \mathrm{~s}$ MF pulses of $5 \mathrm{~Hz}, 26 \mathrm{mT}$ are marked in gray boxes. Shaded area in fluorescence traces is standard error. 

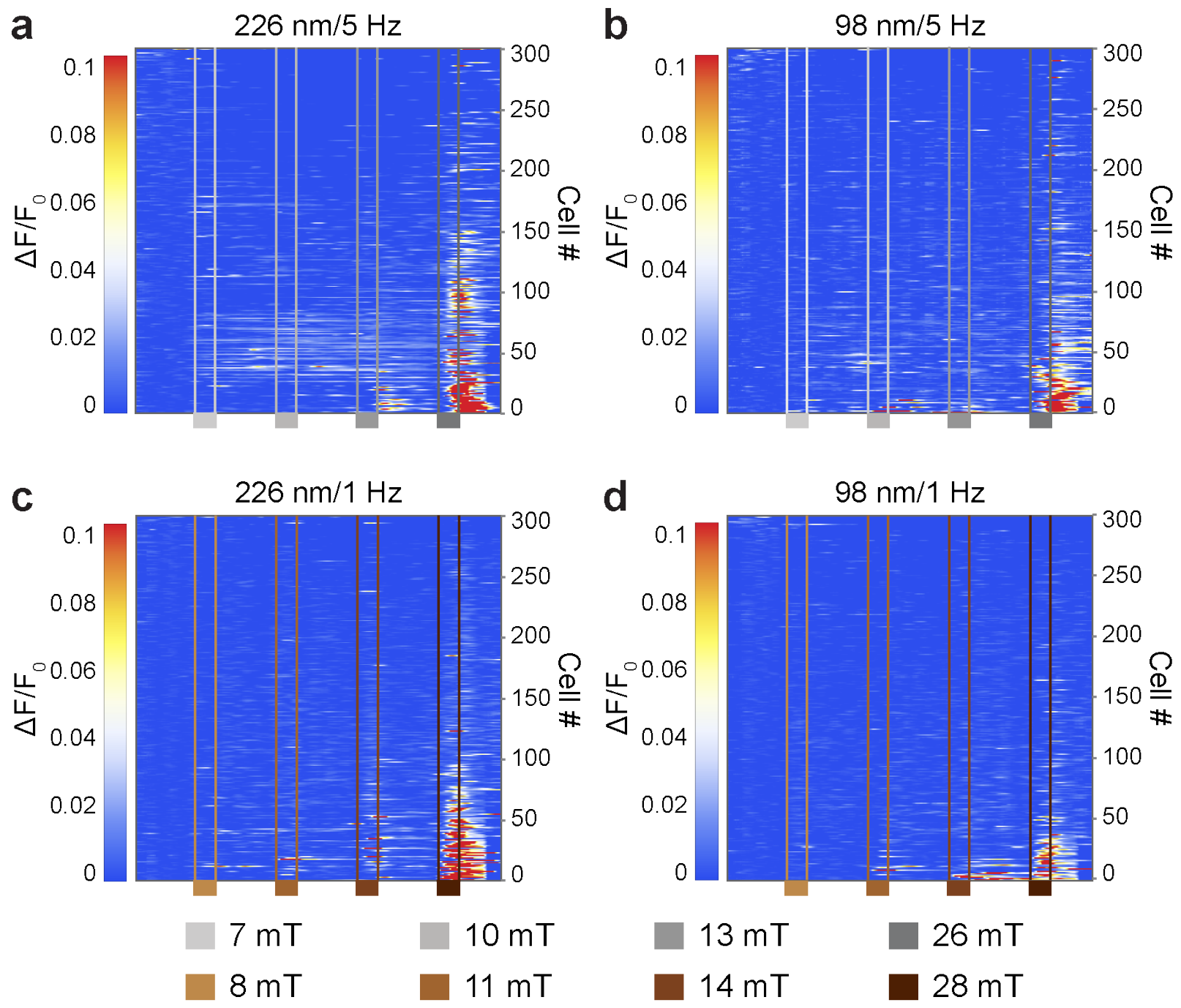

Supplementary Figure 8: Heat maps resulting from DRG $\mathrm{Ca}^{2+}$ imaging for varying MND sizes and MF parameters. This figure corresponds to fluorescence traces and number of activated cells in main Figure 5a. a, MF frequency of $5 \mathrm{~Hz}$ is applied to DRGs decorated with $226 \mathrm{~nm}$ MNDs. b, $5 \mathrm{~Hz}$ MF frequency is applied to the DRGs decorated with $98 \mathrm{~nm}$ MNDs. c, MF frequency of $1 \mathrm{~Hz}$ is applied to the DRGs decorated with $226 \mathrm{~nm}$ MNDs and d, MF frequency of $1 \mathrm{~Hz}$ is applied to the DRGs decorated with $98 \mathrm{~nm}$ MNDs. MF amplitude was sequentially increased for stimulation pulses from $7 \mathrm{mT}$ to $28 \mathrm{mT}$ as noted in the shaded boxes/lines. 
a

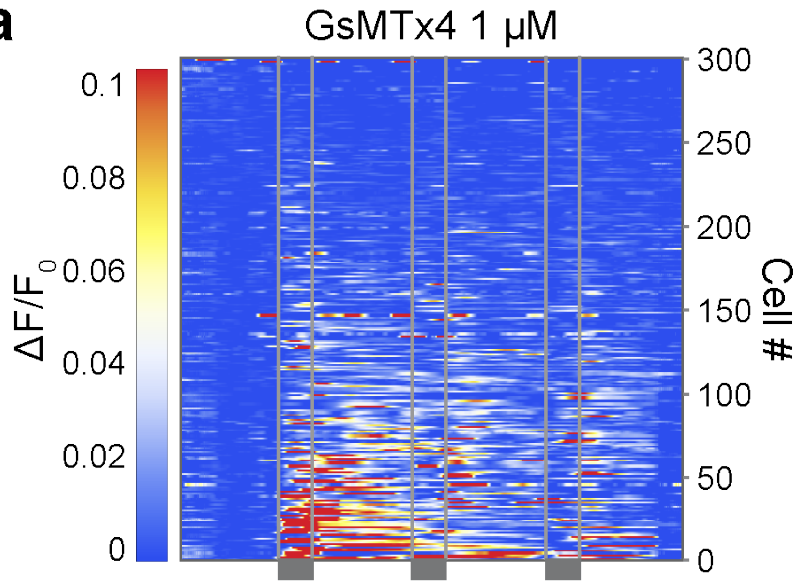

b

b $\quad \mathrm{HC}-0670471 \mu \mathrm{M}$

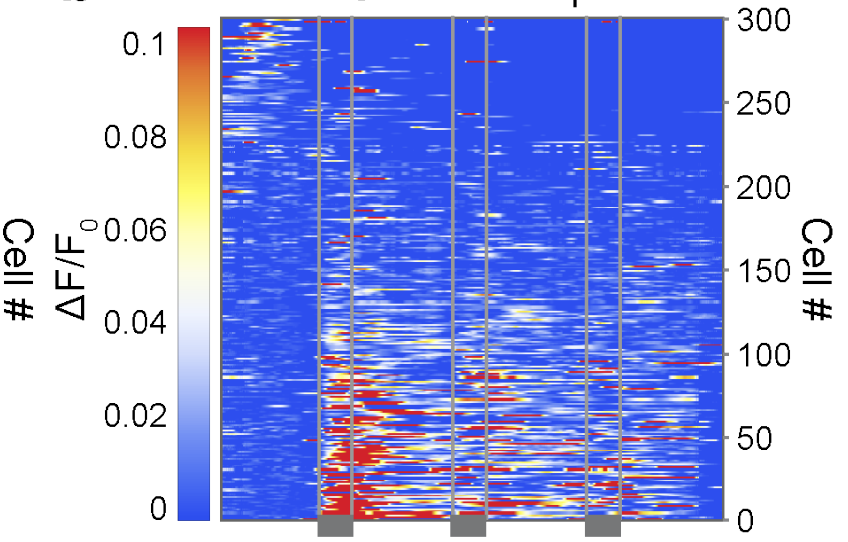

Supplementary Figure 9: $\mathrm{Ca}^{2+}$ imaging for pharmacology experiments during magnetomechanical stimulation with MF of $5 \mathrm{~Hz}$ and $26 \mathrm{mT}$. Figures correspond to the fluorescence traces in main Figure 5c and 5e. a, DRGs incubated with $1 \mu \mathrm{M}$ PIEZO inhibitor GsMTx4 show decrease in activity after first stimulation sequence. $\mathbf{b}$, The same decrease in DRG activity is observed in the case of inhibition with $1 \mu \mathrm{M}$ of TRPV antagonist, HC-067047. 

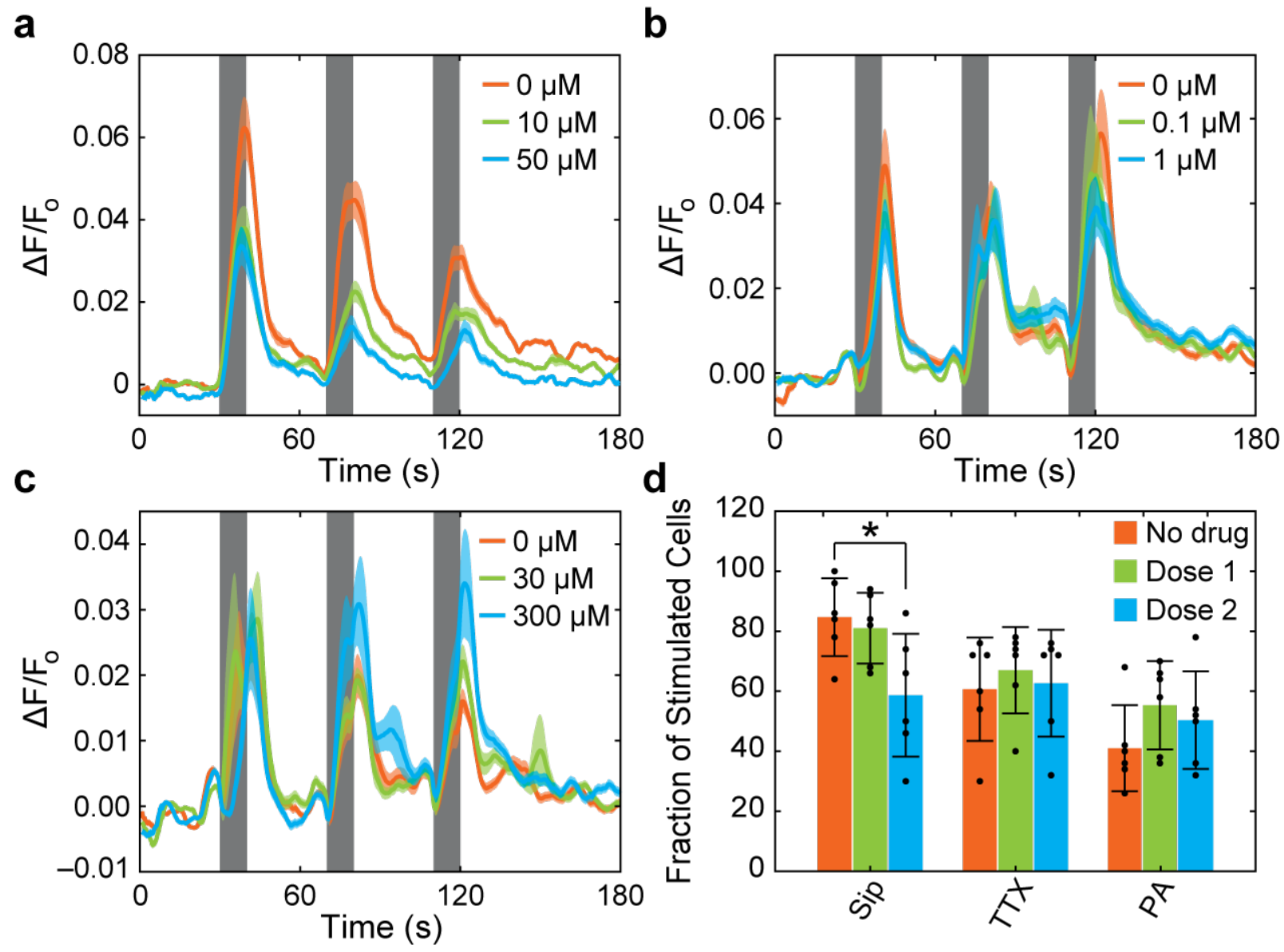

Supplementary Figure 10: Inhibition of sodium and potassium ion channels in DRGs during magnetomechanical stimulation. a, Fluo-4 fluorescence traces for different concentrations of sipatrine (Sip) added to DRGs before magnetomechanical stimulation to inhibit voltage-gated sodium channels and mechanosensitive $\mathrm{K}^{+}$channels TREK-1 and TRAAK. Fluo-4 fluorescence traces for $\mathbf{b}$, tetrodotoxin (TTX) and $\mathbf{c}$, palmitoleic acid (PA) used to inhibit sodium channels and gap junctions in glial cells. $5 \mathrm{~Hz}, 26 \mathrm{mT}$ MF applied in $10 \mathrm{~s}$ epoch as indicated in grey boxes. d, Summary for pharmacology experiments in a-c during magnetomechanical stimulation with 226 $\mathrm{nm}$ diameter MNDs. A significantly lower fraction of cells responds to magnetomechanical stimulation when incubated with $50 \mu \mathrm{M}$ sipatrigine as confirmed by one-way ANOVA ( $\mathrm{n}=6$, $F_{2,15}=4.9, p=0.023$ ) and Tukey's HSD test (tested at $p<0.05$ ). No significant difference in the number of responders was observed for DRGs incubated with TTX or PA, as confirmed by oneway ANOVA $\left(\mathrm{n}=6, F_{2,15}=0.2, p=0.80 ; \mathrm{n}=6, F_{2,15}=1.4, p=0.28\right.$; respectively). Shaded area in fluorescence traces represents standard error. 


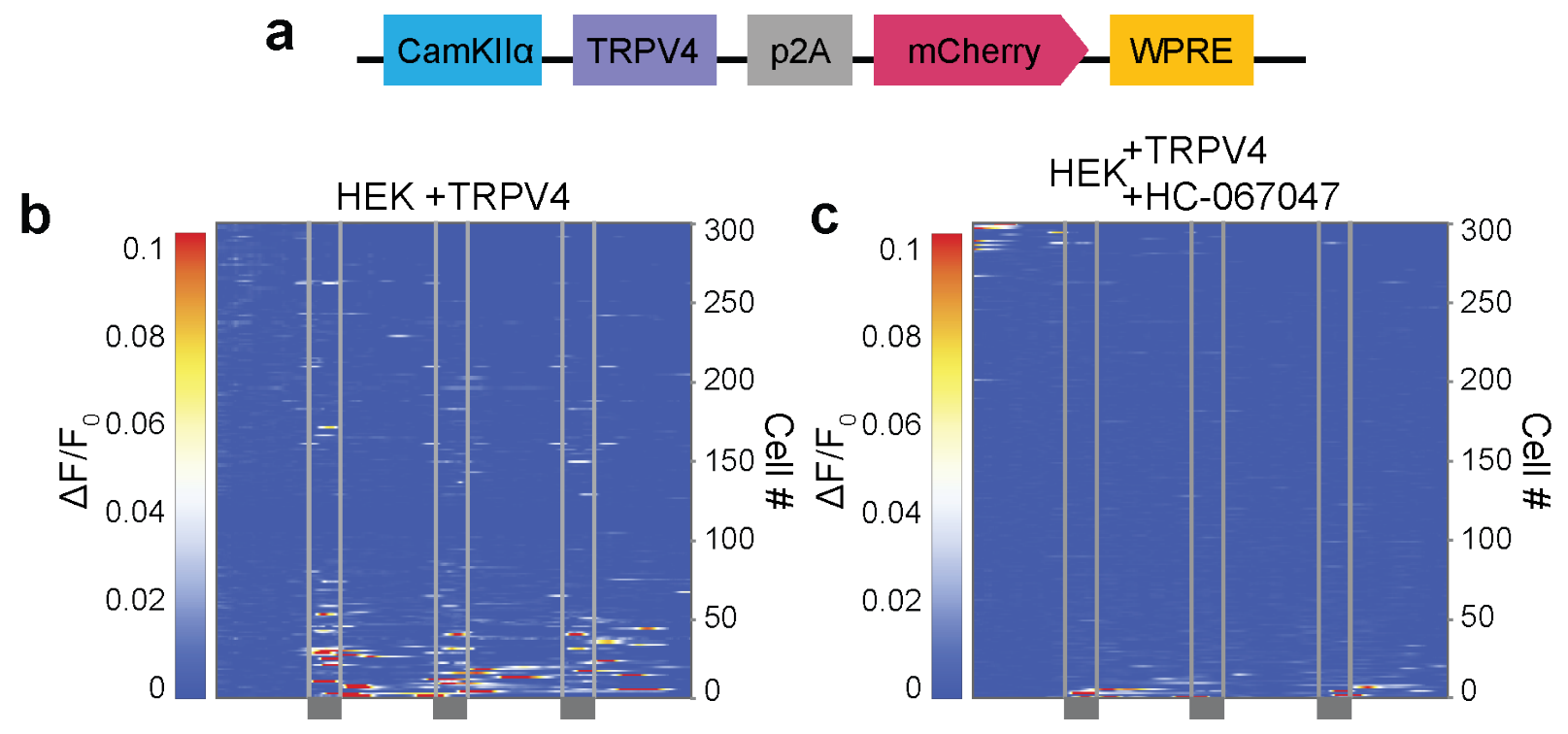

Supplementary Figure 11: MND-mediated activation of HEK-293 cells expressing a mechanosensitive ion channel TRPV4. a, Construct design for delivery of TRPV4 to HEK-293 cells. b, Activity of HEK-293 expressing TRPV4 decorated with $226 \mathrm{~nm}$ MNDs during magnetomechanical stimulation. c, Activity is blocked with $1 \mu \mathrm{M}$ TRPV4 antagonist, HC-067047. $5 \mathrm{~Hz}, 26 \mathrm{mT}$ MF is applied in 3 sequences of 10 seconds as shown in gray boxes. 

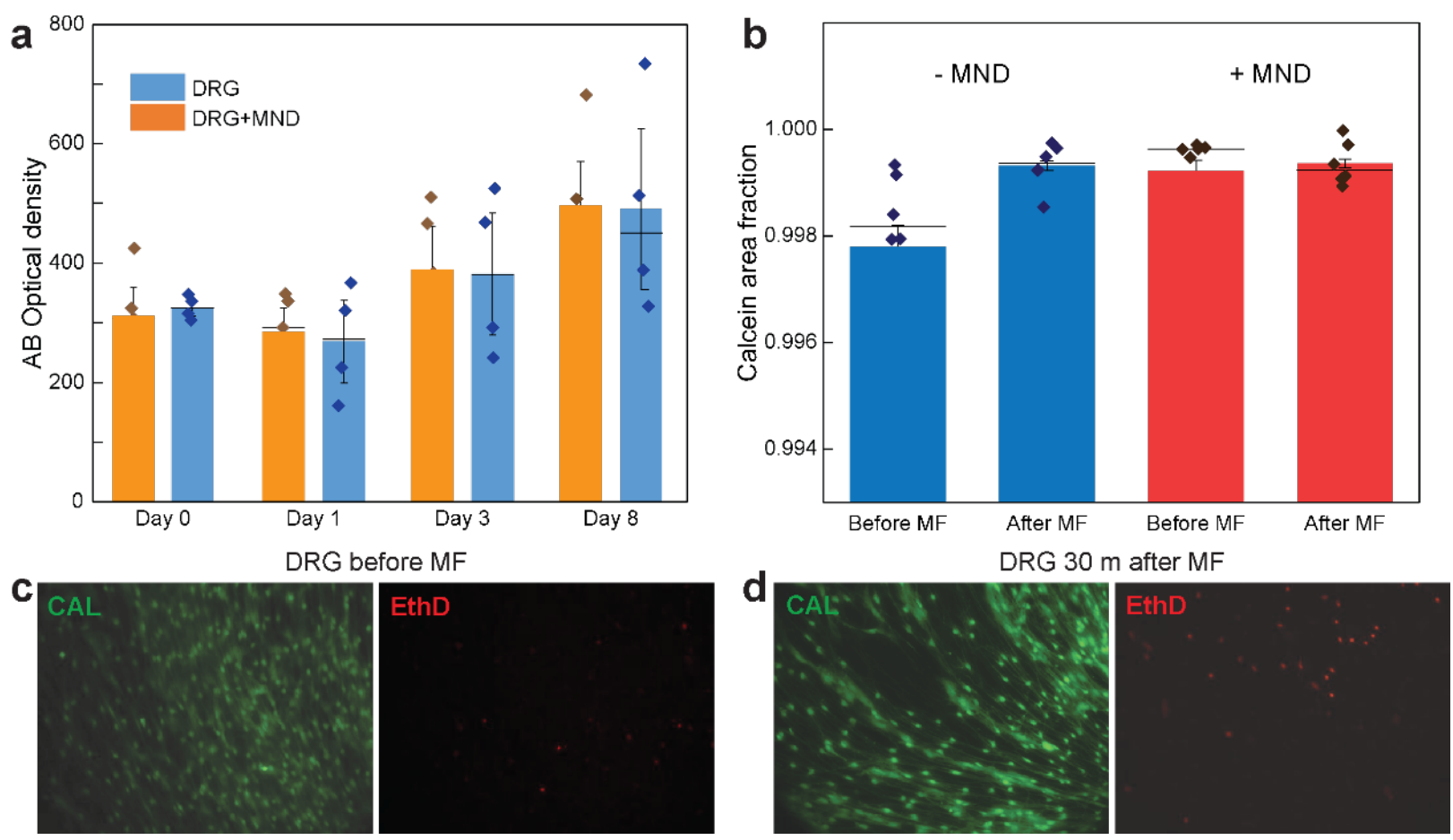

Supplementary Figure 12: Biocompatibility and viability of MNDs on DRG explants. a, Biocompatibility assay determined with alamarBlue for DRGs decorated with $226 \mathrm{~nm}$ diameter MNDs and control DRGs without MNDs. No statistical difference between MND-decorated cells and control cells is observed. b, No significant difference is observed in cell viability after magnetomechanical stimulation of DRGs. Calcein area fraction represents fraction of live cells. c, Live/dead staining of DRGs before mechanostimulation and $\mathbf{d}$, Live/dead cell staining 30 minutes after mechanostimulation. Calcein (CAL) stains live cells in green, ethidium homodimer (EthD) stains dead cells in red. 
Supplementary Tables

\begin{tabular}{|c|c|c|c|c|c|c|}
\hline & 226 DRG & 98 DRG & 226 Hipp & 98 Hipp & - DRG & - Hipp \\
\hline 226 DRG & & 0.17 & $5.8 \times 10^{-4}$ & $6.7 \times 10^{-7}$ & $6.0 \times 10^{-8}$ & $3.5 \times 10^{-8}$ \\
\hline 98 DRG & & & 0.22 & $5.5 \times 10^{-4}$ & $2.7 \times 10^{-5}$ & $9.0 \times 10^{-6}$ \\
\hline 226 Hipp & & & & 0.16 & $1.5 \times 10^{-2}$ & $5.2 \times 10^{-3}$ \\
\hline 98 Hipp & & & & & 0.88 & 0.68 \\
\hline- DRG & & & & & & 1.0 \\
\hline - Hipp & & & & & & \\
\hline
\end{tabular}

Supplementary Table 1: Results of Tukey's honest significant difference test comparing magnetomechanical stimulation results plotted in Figure $4 \mathrm{~h}$. The $p$-values in the table represent the probability of a chance model generating values at least as extreme as those observed, if in that model the means were the same for both conditions (where a condition is, for example, DRGs incubated with $226 \mathrm{~nm}$ nanodiscs). Tukey's honest significant difference test corrects for the Type I error probability increase that comes with multiple comparisons.

\section{Supplementary Movies}

Supplementary Movie S1. Magnetomechanical stimulation evokes calcium activity in DRG explant culture. Side-by-side movies of dorsal root ganglion (DRG) explant culture incubated with Fluo-4 calcium indicator. When magnetic field is applied $(5 \mathrm{~Hz}, 26 \mathrm{mT})$ as indicated by "FIELD ON" in the movie, calcium influx is observed in the DRG incubated with magnetic nanodiscs (right) but not in the DRG without magnetic nanoparticles (left). The movies are at 10X speed.

\section{Data Analysis Scripts}

MATLAB script MovingAverageFilter converts the average grayscale value of each region of interest (a circled cell) over time into $\Delta \mathrm{F} / \mathrm{F}_{0}$ traces with a moving average filter:

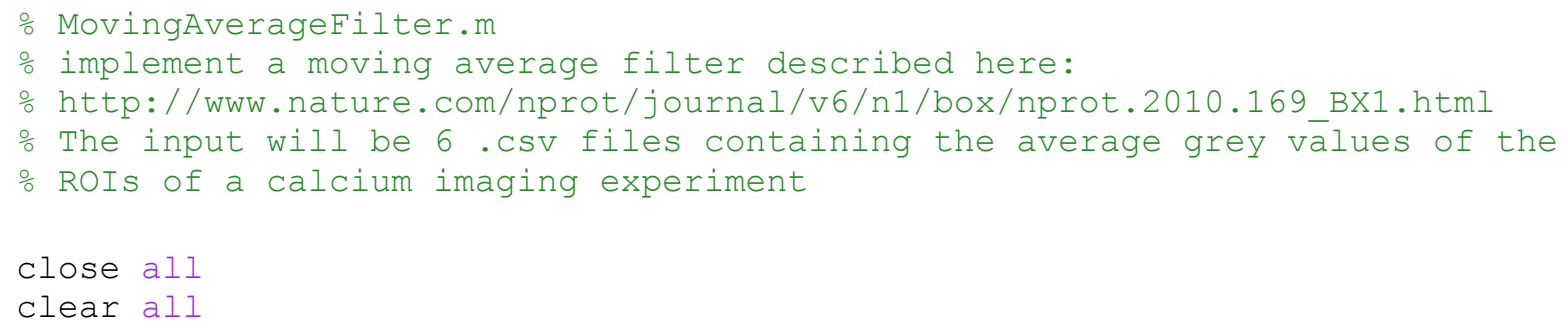




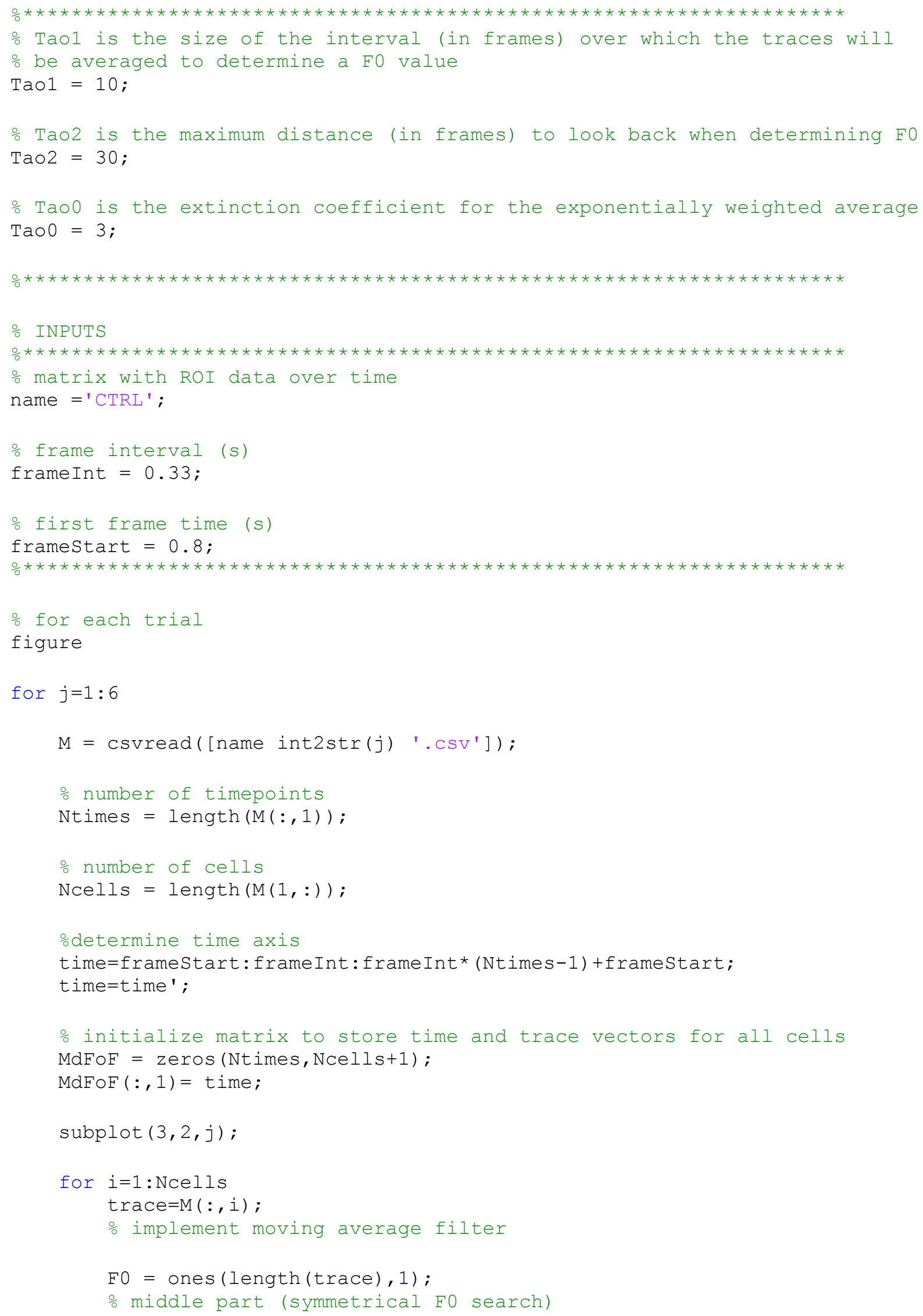




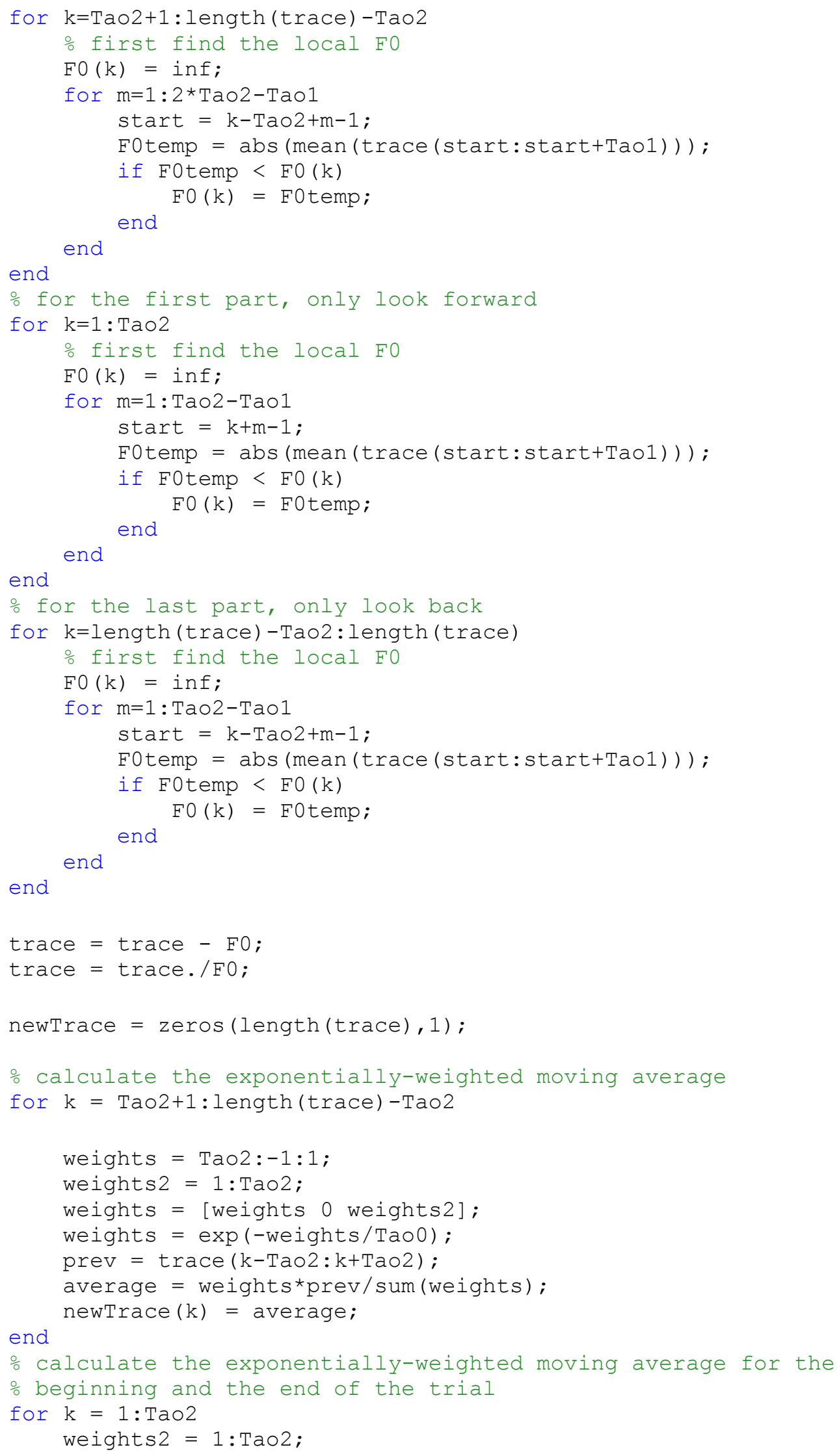




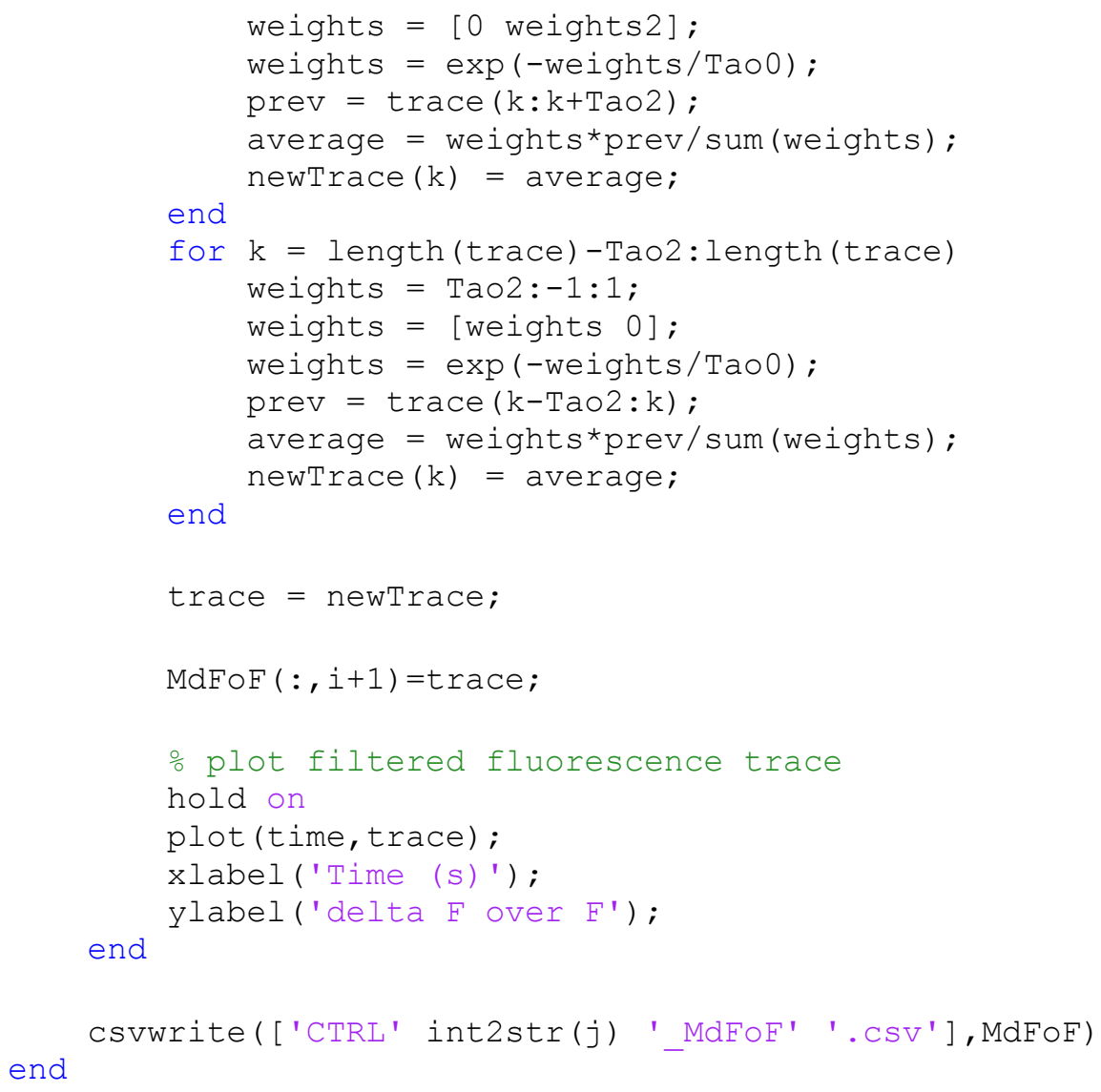

MATLAB script $d F o F \_a n a l y z e r$ selects at random a subset of 50 cells from each of 6 experiments and classifies them as responsive or non-responsive based on whether their $\Delta \mathrm{F} / \mathrm{F}_{0}$ $(\mathrm{dFoF})$ trace ever exceeds 10 times the standard deviation of the signal during the period before MF is ever applied:

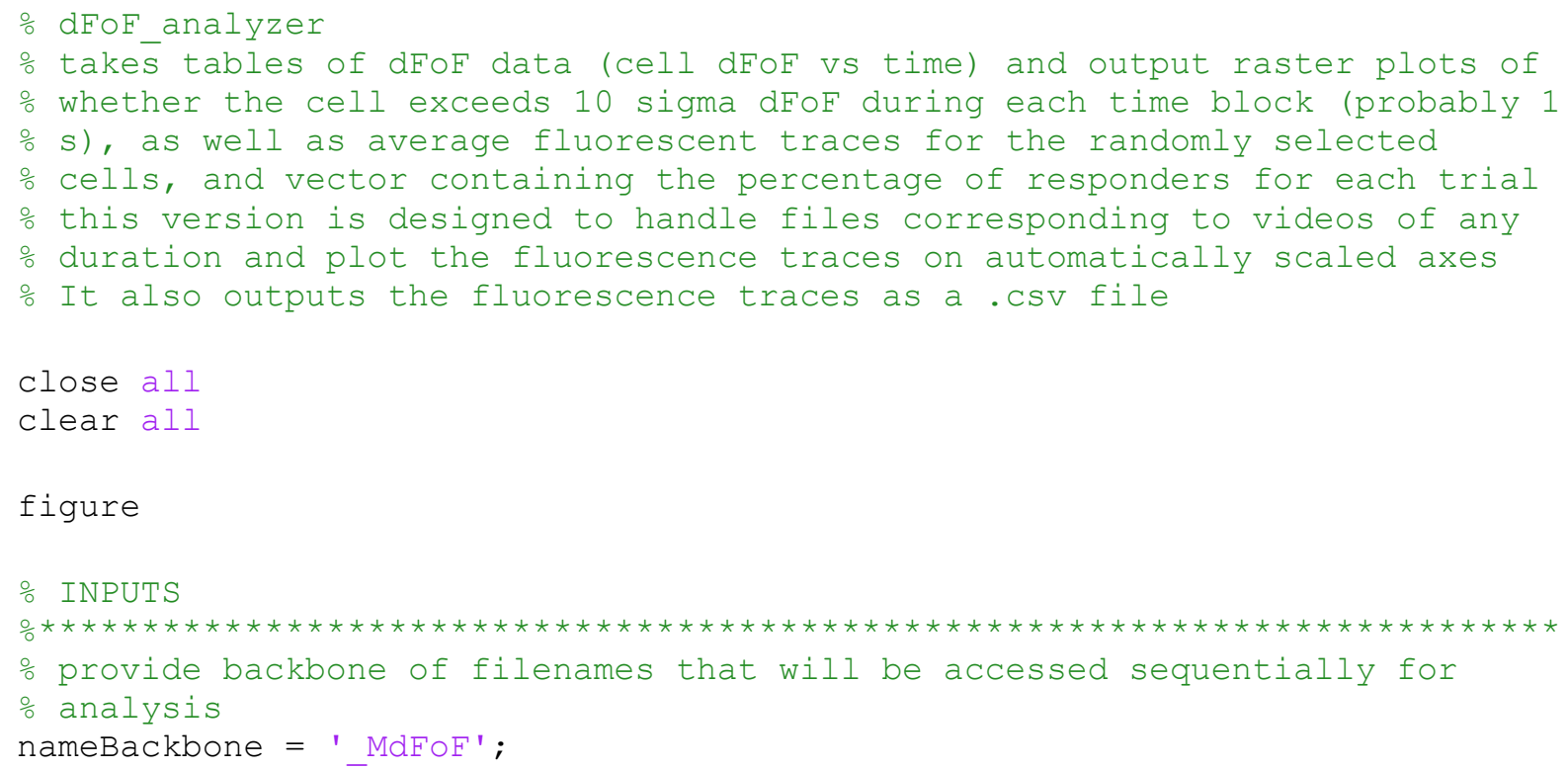




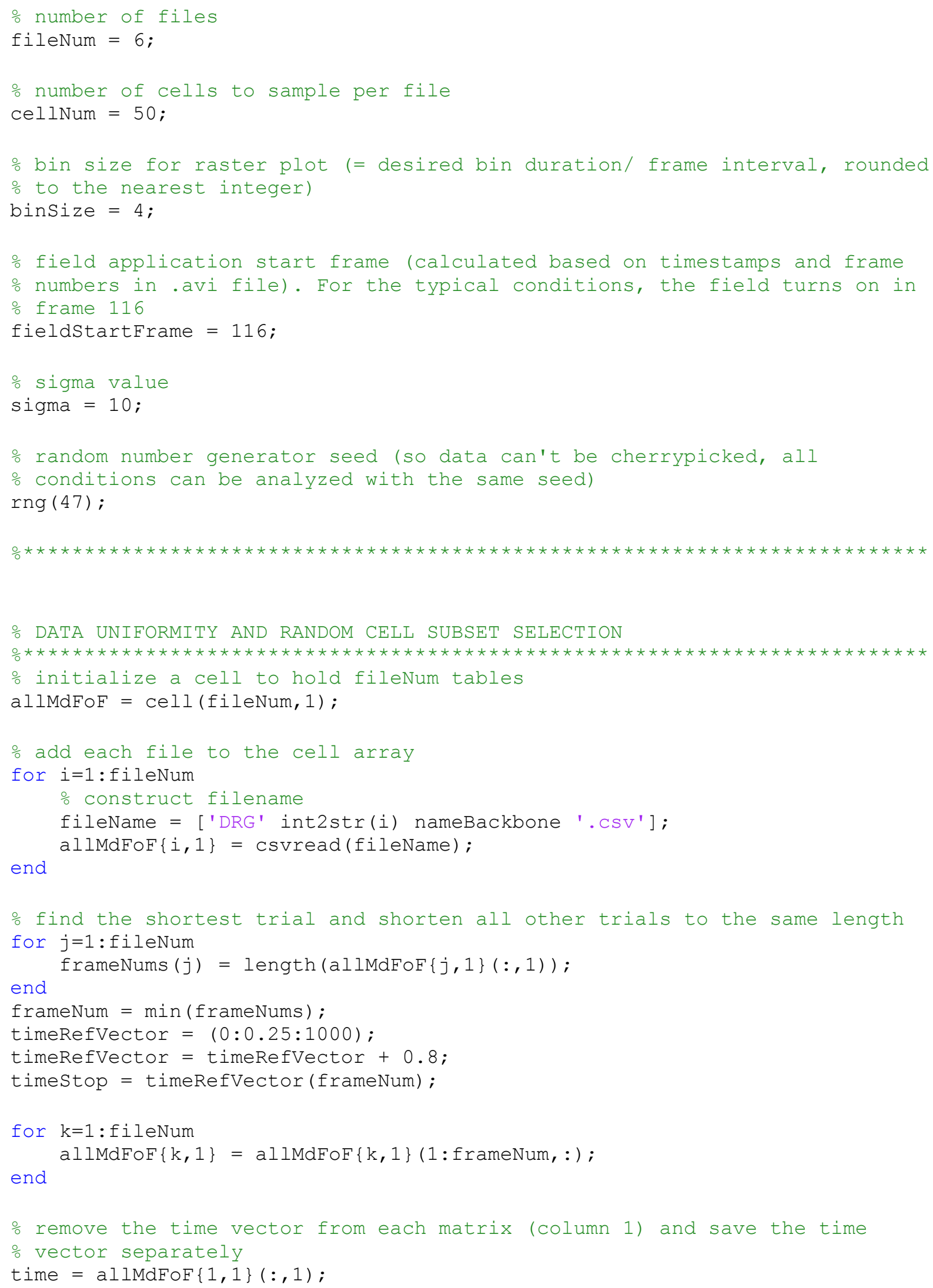




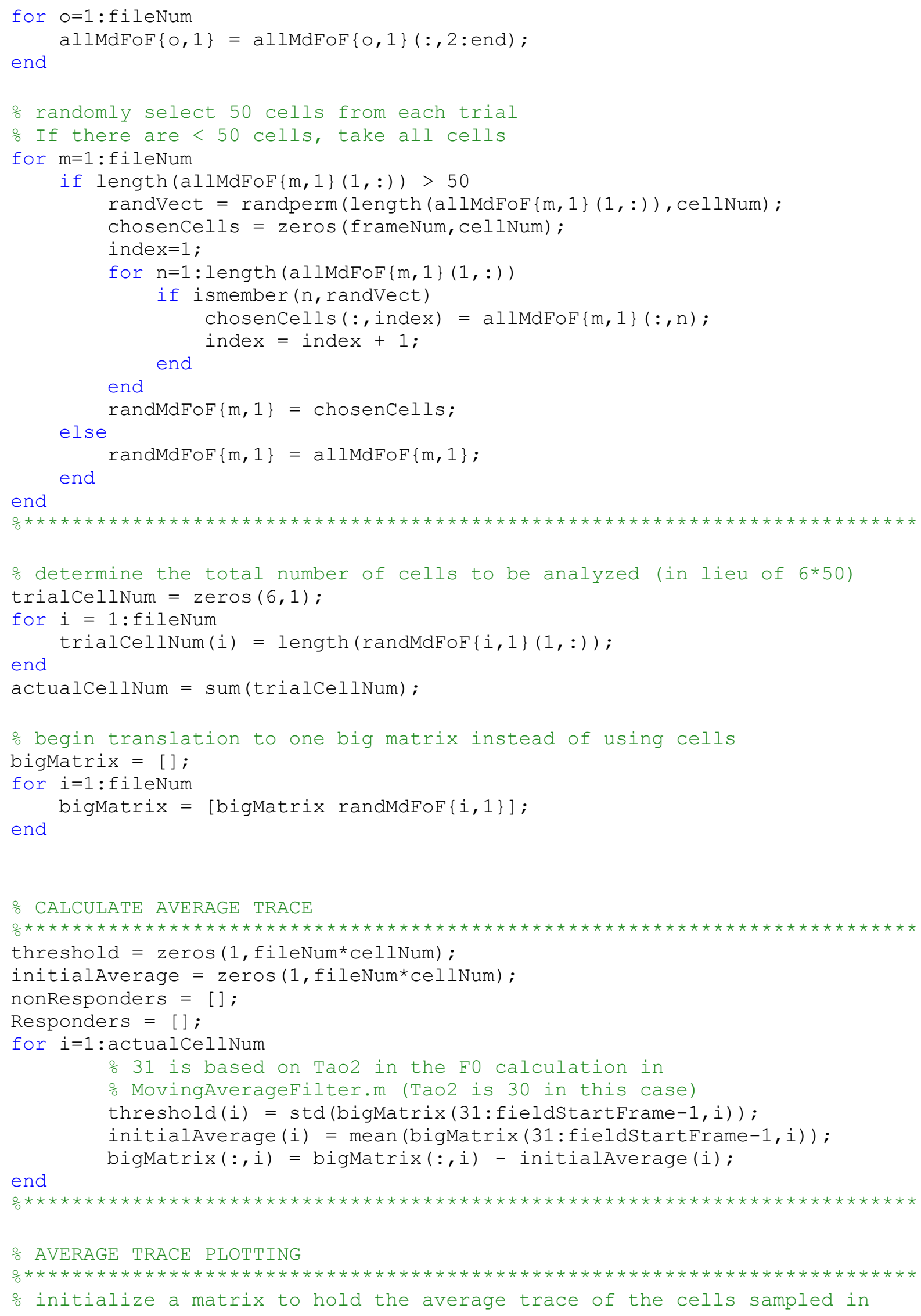


o each trial. The mean and standard deviation of these traces will be

o plotted

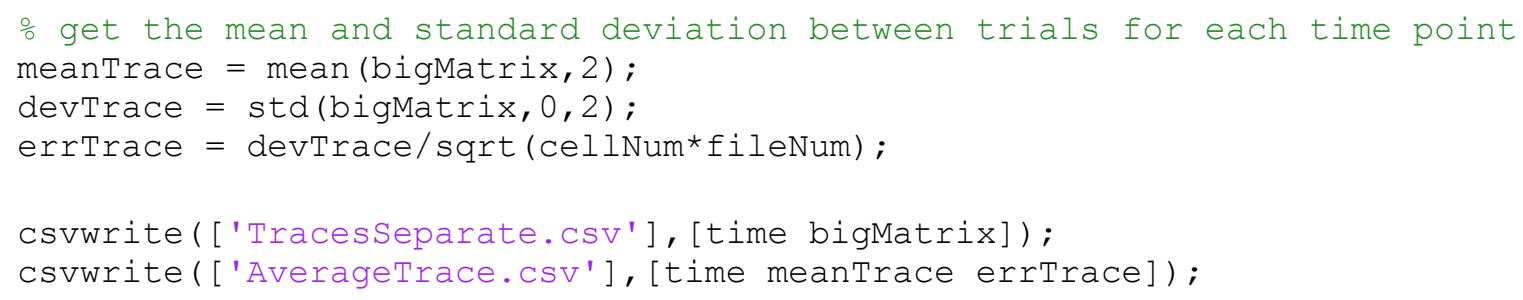




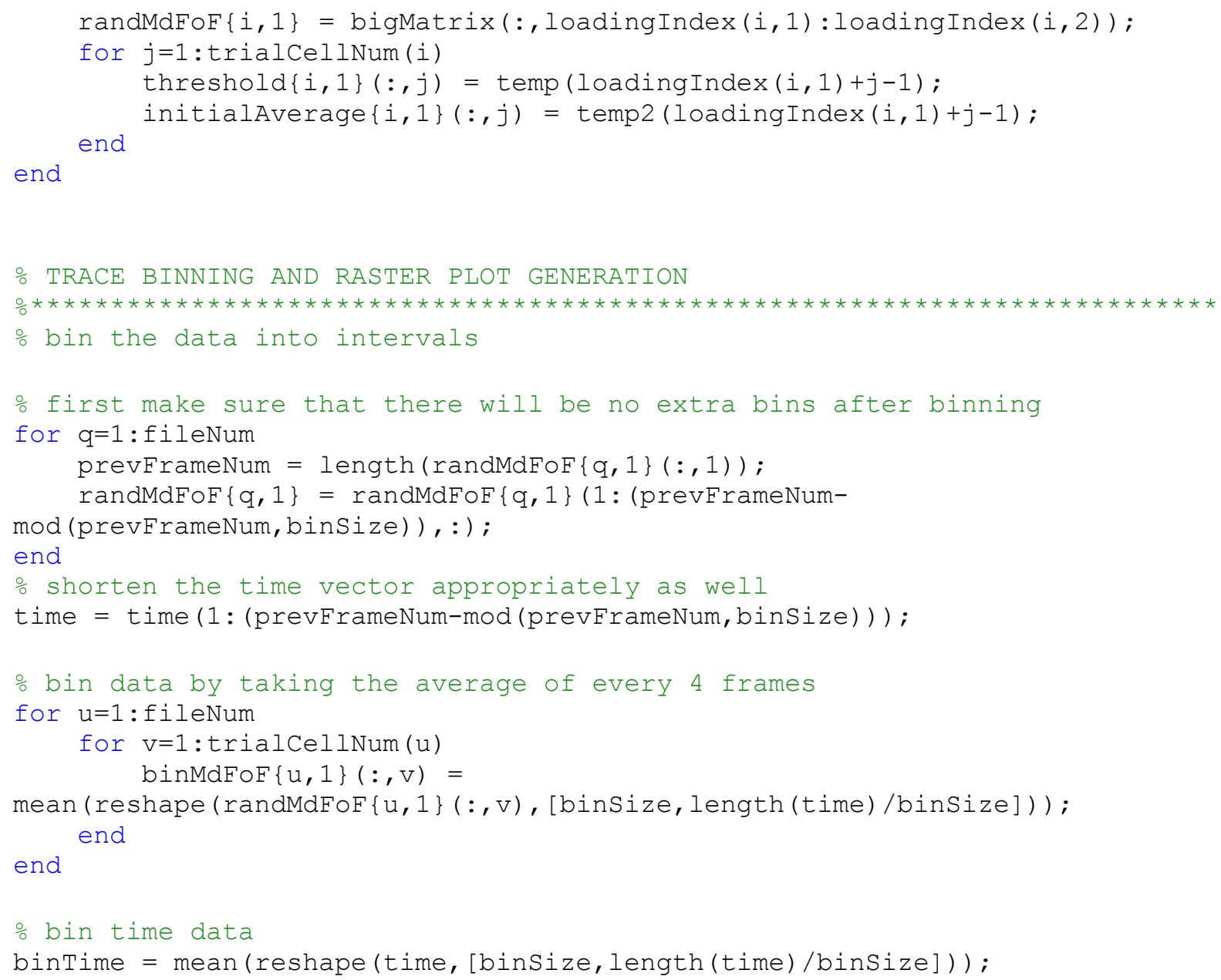




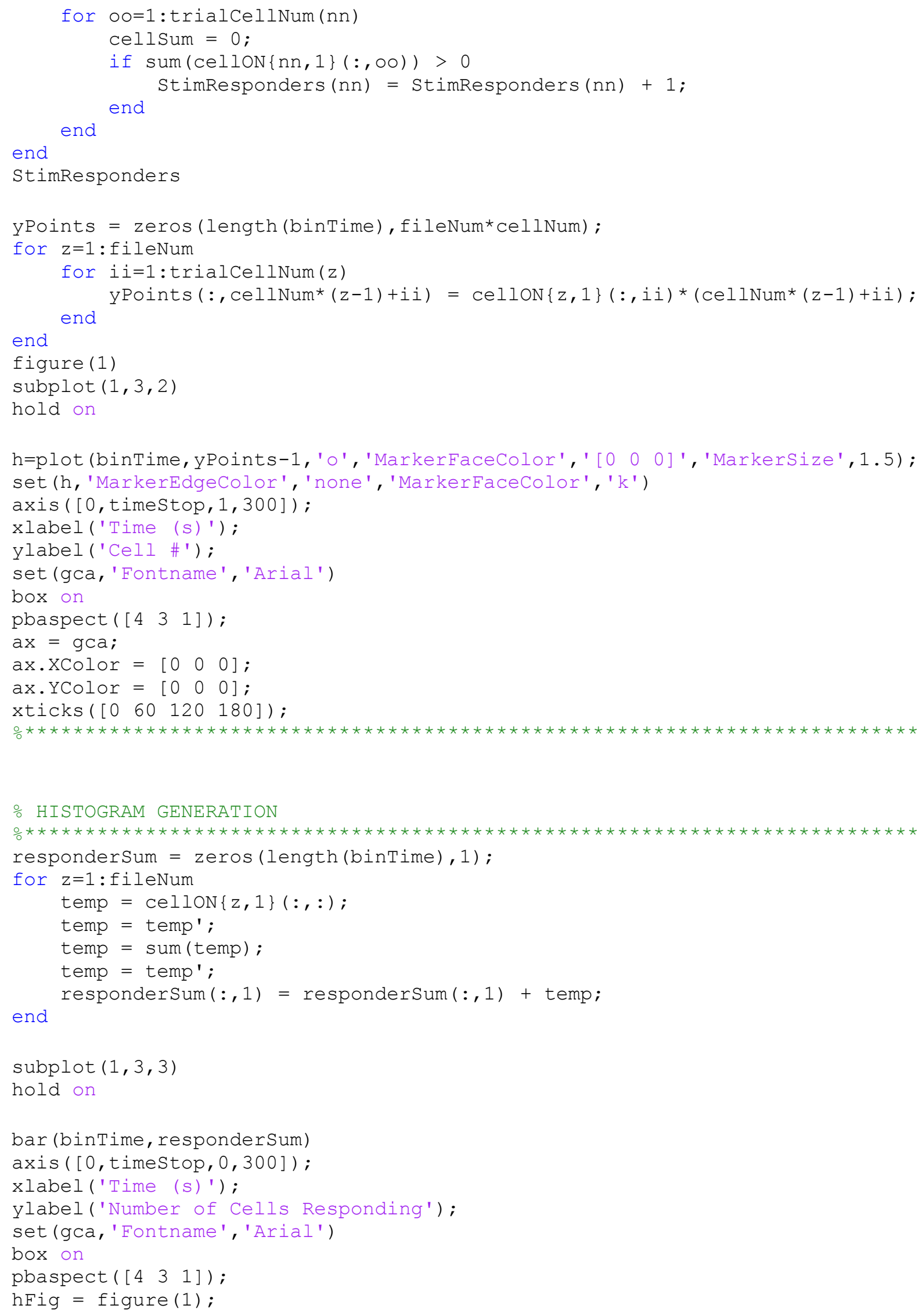


set (hFig, 'Paperorientation', ' landscape') ;

$\mathrm{ax}=\mathrm{gca}$;

ax.XColor $=\left[\begin{array}{lll}0 & 0 & 0\end{array}\right]$;

ax.YColor $=\left[\begin{array}{lll}0 & 0 & 0\end{array}\right]$;

xticks ([0 600120180$])$;

o 